\title{
EL IMPACTO EN LA SALUD MENTAL DE LOS PROFESIONALES SANITARIOS DURANTE LA COVID-19
}

\author{
DAVID BATALLA-MARTÍN ${ }^{1}$, KARINA CAMPOVERDE ESPINOSA² Y MIRIAM BRONCANO-BOLZONI³ \\ ${ }^{1}$ Enfermero de salud mental. Centro de Salud Mental Nou Barris. Barcelona. \\ 2Enfermera especialista en salud mental. Benito Menni CASM. Granollers (Barcelona). \\ ${ }^{3}$ Enfermera de la Red de Salud Mental y Adicciones del Institut d'Assistència Sanitària. Girona.
}

\section{RESUMEN}

Objetivo: examinar cuáles son los impactos psicológicos en la salud mental de los profesionales sanitarios durante el período de la enfermedad por coronavirus de 2019 (COVID-19). Metodología: revisión de bases de datos electrónicas: PubMed, Medline, Web of Science y la base de datos bibliográfica para enfermería CUIDEN, utilizando las palabras clave «COVID-19», «coronavirus», «SARS-CoV-2», «mental health», «nurse», «insomnia», "anxiety», «staff», "health workers» y «review», en trabajos publicados entre diciembre de 2019 y mayo de 2020 y limitada a los idiomas inglés, español y catalán. Resultados: de los 22 artículos incluidos en la revisión, mayoritariamente, se identificaron cuatro impactos psicológicos en los profesionales sanitarios: ansiedad, depresión, insomnio y estrés. Asimismo, se identificaron los factores de riesgo asociados a cada uno de los impactos psicológicos. Conclusiones: esta situación sanitaria provocada por la COVID-19 ha producido importantes impactos psicológicos en los profesionales sanitarios, siendo necesario no solo su detección, sino también su tratamiento para evitar posibles problemas a medio-largo plazo.

Palabras clave: COVID-19, coronavirus, SARS-CoV-2, mental health, nurse, insomnia, anxiety, staff, health workers, review.

\section{INTRODUCCIÓN}

En diciembre de 2019 surgió en Wuhan, China, el brote de una nueva infección causada por el virus SARS-CoV-2, conocido como COVID-19 (enfermedad

Correspondencia: David Batalla Martín Correo electrónico: david.batalla@csm9b.com por coronavirus de 2019; por el acrónimo en inglés de: coronavirus disease-2019), que experimentó una expansión rápida por el resto de los continentes ${ }^{1}$. En España, el primer caso de COVID-19 se notificó en Palma de Mallorca, con una veloz diseminación por todo el país, siendo necesario declarar la situación como una emergencia de salud pública. Desde el inicio de la alerta por COVID-19 hasta el 29 de mayo 
de 2020, se han notificado 250273 casos en España². La distribución por sexo y grupo de la enfermedad se sitúa entre los mayores de 50 años, tanto en hombres como en mujeres².

Esta situación crítica sobrecargó a los sistemas de salud e incrementó el trabajo de los profesionales sanitarios, quienes se encuentran en primera línea y en contacto directo con los pacientes con COVID-19. Además del aumento de la carga de trabajo, el trabajar bajo presiones extremas, tomar decisiones difíciles a nivel ético, aumentar las horas en los turnos de trabajo y la preocupación por la transmisión nosocomial al estar expuestos continuamente a pacientes con COVID-19 hace que el bienestar físico y mental de los trabajadores sanitarios pueda verse afectado a medio-largo plazo.

Estudios anteriores relacionados con el síndrome respiratorio agudo grave (SARS; por las siglas en inglés de: severe acute respiratory syndrome) en 2003 mostraron resultados de impacto psicológico en profesionales sanitarios con el riesgo de desarrollar ansiedad, depresión, estrés postraumático u otros síntomas de salud mental ${ }^{3-5}$. Motivo por el cual se plantea el objetivo de examinar cuáles son esas repercusiones psicológicas en la salud mental de los profesionales sanitarios durante el brote de COVID-19 y los factores que pueden influir en dicho impacto psicológico.

\section{MATERIAL Y MÉTODO}

\section{Búsqueda de artículos}

Se llevó a cabo una búsqueda de artículos en las siguientes bases de datos electrónicas: PubMed, Medline, Web of Science y la base de datos bibliográfica para enfermería CUIDEN. Como palabras clave, se introdujeron "COVID-19», "coronavirus», «SARS-CoV-2», «mental health», «nurse», «insomnia», «anxiety», «staff», «health workers» y «review». La búsqueda cubre el período de diciembre de 2019 hasta mayo de 2020.
Los criterios de inclusión de la muestra fueron artículos que analizaran el impacto psicológico sobre la salud mental de los profesionales sanitarios durante la crisis epidémica de la COVID-19. Se aceptaron para su posterior análisis artículos originales, preprints, revisiones, metanálisis y cartas al editor. Los artículos que no se centraban en las repercusiones en el personal sanitario fueron rechazados, como tampoco se aceptaron artículos sobre el impacto psicológico de otras epidemias como la del SARS o la del síndrome respiratorio de Oriente Medio (MERS; por las siglas en inglés de: Middle East respiratory syndrome).

\section{Características de la muestra}

Los artículos obtenidos según los criterios de inclusión y exclusión fueron 22, de los cuales, 17 ya están publicados y 5 se encuentran pendientes de publicación. La metodología de investigación de la mayoría de los artículos - 18 concretamente- fueron análisis cuantitativos, 2 fueron estudios cualitativos y 2 fueron revisiones. Respecto a la procedencia de los estudios incluidos en nuestra revisión, mayoritariamente se han realizado en países asiáticos: China (14 estudios), Taiwán (1 estudio), Singapur (1 estudio) y Shanghái (1 estudio). De los 5 restantes, los estudios fueron realizados en Italia (1 estudio), Arabia Saudí (1 estudio), Barcelona (1 carta al editor), Reino Unido y Brasil (2 revisiones). La muestra estudiada estaba compuesta de, al menos, 18 y un máximo de 11118 personas. Mayoritariamente el personal sanitario analizado estaba formado por enfermeras y médicos, aunque también se tuvieron en cuenta otros perfiles sanitarios como los de auxiliares de enfermería, técnicos y estudiantes de enfermería.

\section{Evaluación de los artículos}

Todos los artículos seleccionados por parte de los investigadores fueron analizados para conocer la metodología de análisis, las variables analizadas sobre Ios efectos de la COVID-19 respecto a la salud 
mental de los profesionales sanitarios, las escalas y procedimientos estadísticos utilizados para su estudio, los resultados, los hallazgos principales, las im- plicaciones futuras y sus limitaciones. Los artículos, cartas a los editores y revisiones seleccionadas y analizadas pueden verse en detalle en la tabla 1 .

Tabla 1. Artículos, cartas a los editores y revisiones seleccionadas

\begin{tabular}{|c|c|c|}
\hline Título de artículo & Autores & Provincia, país \\
\hline $\begin{array}{l}\text { «Factors associated with mental health outcomes among health } \\
\text { care workers exposed to coronavirus disease } 2019 »\end{array}$ & Lai et al. ${ }^{3}$ & $\begin{array}{l}\text { Wuhan, Hubéi, } \\
\text { China }\end{array}$ \\
\hline $\begin{array}{l}\text { "COVID-19 in Wuhan: immediate psychological impact on } 5062 \\
\text { health workers» }\end{array}$ & Zhu et al. ${ }^{6}$ & Wuhan, China \\
\hline $\begin{array}{l}\text { «Covid-19 pandemic impact on mental health: a web-based } \\
\text { cross-sectional survey on a sample of Italian general } \\
\text { practitioners» }\end{array}$ & Amerio et al. ${ }^{7}$ & Génova, Italia \\
\hline $\begin{array}{l}\text { "COVID-19-related information sources and psychological } \\
\text { well-being: an online survey study in Taiwan» }\end{array}$ & Ko et al. ${ }^{8}$ & Taiwán \\
\hline $\begin{array}{l}\text { «A comparison of burnout frequency among oncology physicians } \\
\text { and nurses working on the frontline and usual wards during the } \\
\text { COVID-19 epidemic in Wuhan, China» }\end{array}$ & Wu et al. ${ }^{9}$ & Wuhan, China \\
\hline $\begin{array}{l}\text { «Mental health and psychosocial problems of medical health } \\
\text { workers during the COVID-19 epidemic in China» }\end{array}$ & Zhang et al. ${ }^{10}$ & China \\
\hline $\begin{array}{l}\text { «Mental health status of doctors and nurses during COVID-19 } \\
\text { epidemic in China» }\end{array}$ & Liu et al. ${ }^{11}$ & China \\
\hline $\begin{array}{l}\text { «Prevalence of depression, anxiety, and insomnia among } \\
\text { healthcare workers during the COVID-19 pandemic: a systematic } \\
\text { review and meta-analysis» }\end{array}$ & Pappa et al. ${ }^{12}$ & Reino Unido \\
\hline $\begin{array}{l}\text { «Psychological effects of COVID-19 on hospital staff: a national } \\
\text { cross-sectional survey of China Mainland» }\end{array}$ & Guo et al. ${ }^{13}$ & China \\
\hline $\begin{array}{l}\text { «Psychological impact of the COVID-19 pandemic on health care } \\
\text { workers in Singapore» }\end{array}$ & Tan et al. ${ }^{14}$ & Singapur \\
\hline $\begin{array}{l}\text { "Psychological status of medical workforce during the COVID-19 } \\
\text { pandemic: a cross-sectional study» }\end{array}$ & Lu et al. ${ }^{15}$ & Fujián, China \\
\hline $\begin{array}{l}\text { «Psychological status of surgical staff during the COVID-19 } \\
\text { outbreak» }\end{array}$ & $\mathrm{Xu}$ et al. ${ }^{16}$ & Shanghái, China \\
\hline $\begin{array}{l}\text { «Psychological symptoms among frontline healthcare workers } \\
\text { during COVID-19 outbreak in Wuhan» }\end{array}$ & Du et al. ${ }^{17}$ & Wuhan, China \\
\hline $\begin{array}{l}\text { «Stressors of nurses in psychiatric hospitals during the COVID-19 } \\
\text { outbreak» }\end{array}$ & Wu et al. ${ }^{18}$ & Chengdú, China \\
\hline $\begin{array}{l}\text { «Survey of insomnia and related social psychological factors } \\
\text { among medical staff involved in the } 2019 \text { novel coronavirus } \\
\text { disease outbreak» }\end{array}$ & Zhang et al. ${ }^{19}$ & China \\
\hline
\end{tabular}


Tabla 1. Artículos, cartas a los editores y revisiones seleccionadas (cont.)

\section{Título de artículo}

"The effects of social support on sleep quality of medical staff treating patients with coronavirus disease 2019 (COVID-19) in January and February 2020 in China»

«The evaluation of sleep disturbances for Chinese frontline medical workers under the outbreak of COVID-19»

"The prevalence and influencing factors in anxiety in medical workers fighting COVID-19 in China: a cross-sectional survey»

«The psychological impact of COVID-19 pandemic on health care workers in a MERS-CoV endemic country»

«When health professionals look death in the eye: the mental health of professionals who deal daily with the 2019 coronavirus outbreak»

«Work stress among Chinese nurses to support Wuhan in fighting against COVID-19 epidemic»

«Resilience and emotional support in health care professionals during the COVID-19 pandemic»

\begin{tabular}{|c|c|}
\hline Autores & Provincia, país \\
\hline Xiao et al. ${ }^{20}$ & \multicolumn{1}{|c|}{ China } \\
\hline Qi et al. ${ }^{21}$ & China \\
\hline Liu et al. ${ }^{22}$ & China \\
\hline Temsah et al. ${ }^{23}$ & Arabia Saudí \\
\hline Rolim Neto et al. ${ }^{24}$ & Brasil \\
\hline Mo et al. ${ }^{25}$ & China \\
\hline Frías et al. ${ }^{26}$ & Barcelona, España \\
\hline
\end{tabular}

\section{RESULTADOS}

Los artículos seleccionados para esta revisión se centran en analizar cómo ha sido el impacto psicológico de los profesionales que han intervenido en la atención clínica durante la crisis sanitaria de la COVID-19, pero no únicamente en aquellos profesionales sanitarios que han estado en primera línea durante el período más crítico, sino también en aquellos sanitarios que han trabajado en otras unidades o en programas comunitarios. Estos estudios, mayoritariamente, han coincidido en el análisis de este impacto psicológico desde cuatro síntomas clínicos: la ansiedad, el estrés, la depresión y el insomnio. Otros síntomas y/o conceptos que han sido analizados también por los autores de estos artículos han sido la calidad de vida, el burnout, la resiliencia, la salud global, el miedo y la satisfacción personal7 $7,9,15,16,18,26$, pero estos no han estado presentes en la mayoría de los artículos.

A continuación, detallamos los principales impactos psicológicos respecto a los síntomas coincidentes.

\section{Ansiedad}

Se han encontrado 17 estudios en los que la ansiedad aparecía como uno de los principales impactos psicológicos analizados en los profesionales sanita$\operatorname{rios}^{3,6,7,10,12-17,19,20,22-25}$.

Para evaluar el impacto de la ansiedad en los profesionales sanitarios, los diferentes autores utilizaron los siguientes cuestionarios estandarizados: la Escala para el Trastorno de Ansiedad Generalizada (Generalized Anxiety Disorder, GAD-7); la Escala de Ansiedad de Hamilton (Hamilton Anxiety Scale, HAMA); la Escala de Autoevaluación de la Ansiedad de Zung (Zung Self-rating Anxiety Scale, SAS); el Inventario

\section{Cuatro síntomas clínicos: la ansiedad, el estrés, la depresión y el insomnio.}


de Ansiedad de Beck (Beck Anxiety Inventory, BAI); y las Escalas de Depresión, Ansiedad y Estrés (Depresion, Anxiety and Stress Scales, DASS-21).

En los estudios encontrados, se puede observar que mayoritariamente se emplearon la escala GAD-7 y la SAS. Los resultados obtenidos por medio de la GAD-7 mostraron en el estudio de Lai et al. ${ }^{3}$ que el $44,6 \%$ presentaban ansiedad. La muestra estudiada estaba compuesta por 1257 participantes, donde el $76,7 \%$ eran mujeres. Resultados similares encontramos en el estudio de Zhang et al. ${ }^{19}$, en el cual la ansiedad representaba el 44,7\% dentro de una muestra de 1563 participantes, siendo el $75 \%$ mujeres.

Los resultados obtenidos por medio de la escala SAS muestran que el 4,98\% presentan niveles de ansiedad medios y altos en los profesionales sanitarios en una muestra compuesta por 11118 participantes ${ }^{13}$. En el estudio de Liu et al. ${ }^{11}$, la ansiedad tiene una prevalencia del $16 \%$ en una muestra de 4679 médicos y enfermeras de 348 hospitales de China. Finalmente, el estudio de Mo et al.25 analiza el estrés y la ansiedad mediante la correlación de Pearson y concluye lo siguiente: cuanto mayor es la carga de estrés, mayor será la puntuación de ansiedad.

En el estudio de Tan et al. ${ }^{14}$, se emplearon las escalas DASS-21, dando como resultado una prevalencia de ansiedad en torno al 20,7\% en el personal sanitario no médico frente al $10,8 \%$ del personal sanitario médico. Estos resultados nos muestran que existe mayor riesgo de sufrir trastornos psicológicos dentro del grupo del personal sanitario no médico.

\section{Depresión}

El impacto de la depresión en los profesionales sanitarios durante el brote de COVID-19 se evaluó en $12^{3,6,7,10-17,19}$ de los 22 estudios que se han incorporado en la revisión.

Para evaluar la depresión, se han utilizado los siguientes instrumentos estandarizados: el Inventario de Depresión de Beck (Beck Depression Inventory-II, BDI-II); las DASS-21; la Escala de Depresión de Hamilton (Hamilton Depression Scale, HAMD); el Cuestionario sobre Salud del Paciente-9 (Patient Health Questionnaire 9, PHQ-9); el Cuestionario sobre Salud del Paciente-4 (Patient Health Questionnaire 4, PHQ4); y la Escala de Autoevaluación de la Depresión de Zung (Zung Self-rating Depression Scale, SDS).

En cuatro de los estudios, emplearon el PHQ-9. En el de Zhu et al. ${ }^{6}$, incluyeron 5062 participantes - entre ellos había médicos, enfermeras y técnicos de Tongji-, detectando una prevalencia de un 13,4\% de síntomas de depresión (PHQ-9 $\geq 10$ ). En el estudio de Lai et al. ${ }^{3}$, una proporción considerable de participantes informaron de síntomas depresivos (50,4\%). Otro de los estudios que utilizaron el PHQ9 fue el de Zhang et al. ${ }^{19}$, donde el 50,7\% presentaba síntomas depresivos ( $P H Q-9 \geq 5$ ). Finalmente, en el estudio de Amerio et al. ${ }^{7}$, con una muestra de 131 médicos de cabecera de Génova (Italia), en el que se comparaba entre síntomas depresivos leves o ausentes (PHQ-9 < 10) y moderados o graves, el 22,9\% de los médicos de cabecera presentaba síntomas depresivos moderados o graves.

La SDS fue utilizada en dos de los estudios. En el primero, la prevalencia de los síntomas depresivos fue del 34,6\% (intervalo de confianza [IC] del $95 \%$ : $33,2-35,9)^{11}$. En el segundo, el de Guo et al. ${ }^{13}$, la prevalencia fue del $28,22 \%$.

La depresión también fue analizada a través del PHQ-4 en una muestra de 2182 participantes de China. De estos, los trabajadores de la salud médicos obtuvieron mayor prevalencia de depresión que los trabajadores de la salud no médicos (del 12,2\% frente al 9,5\% $)^{10}$. Por otro lado, el $12,1 \%$ de una muestra de profesionales sanitarios de China $(n=2042)$ presentaba síntomas depresivos; estos fueron evaluados con la HAMD ${ }^{15}$. Finalmente, Tan et al. ${ }^{14}$ utilizaron las DASS-21 para analizar la depresión, observándose una prevalencia del 8,9\% en una muestra de 470 profesionales sanitarios de Singapur. 


\section{Estrés}

Otro de los impactos psicológicos que destaca en los profesionales sanitarios es el estrés, el cual se evaluó en 13 estudios $3,6,8,8,11,14,17-20,23-25$ de los 22 que se incluyen en esta revisión.

Para evaluar el impacto del estrés en los profesionales sanitarios, los diferentes autores utilizaron las siguientes escalas y cuestionarios estandarizados: la Escala Revisada del Impacto del Suceso (Impact of Event Scale-Revised, IES-R); las DASS-21; la Escala del Estrés Percibido (Perceived Stress Scale, PSS); el Cuestionario de Síntomas (Self-Reporting Questionnaire, SRQ-20); la Escala de Reajuste Social (Social Readjustment Rating Scale, SRRS); y el Cuestionario de Stanford sobre Reacciones ante el Estrés Agudo (Stanford Acute Stress Reaction Questionnaire, SASR).

El estrés laboral representa un motivo de preocupación para los profesionales sanitarios. Así lo demuestra el estudio de Zhang et al. ${ }^{19}$, donde la prevalencia del estrés fue del $73,4 \%$ en una muestra compuesta por 1563 médicos en China. Otro estudio llevado a cabo en Wuhan, China, también analizó la prevalencia del estrés, y se observó que el $54 \%$ de los trabajadores sanitarios de primera línea presentaban niveles moderados o graves de estrés percibido ${ }^{17}$.

En el estudio realizado por Ko et al. ${ }^{8}$, se detectó que el bienestar psicológico quedaba afectado cuando las personas buscaban continuamente información y noticias relacionadas con la COVID-19 para aliviar el estrés. Sobre todo, esta búsqueda de información la realizaban las personas no sanitarias, ya que el personal sanitario mostraba un mejor bienestar psicológico, al tener información oficial sobre la COVID-19.

Asimismo, en el estudio de Tan et al. ${ }^{14}$, al comparar el estrés entre el personal sanitario médico y no médico en Singapur, se detecta que el personal no médico presenta puntuaciones más altas en las escalas DASS-21 e IES-R, aumentando el riesgo de que dicho colectivo sufra mayores trastornos psicológicos durante el período de la COVID-19.

\section{El estrés laboral representa un motivo de preocupación para los profesionales sanitarios.}

Finalmente, también comparan el burnout mediante el Inventario de Burnout de Maslach y Jackson, observando que el desgaste profesional es ligeramente más bajo en el grupo de los profesionales de primera línea en comparación con el de los profesionales que desempeñan su función laboral en sus unidades de referencia habituales ${ }^{18}$.

\section{Insomnio}

Otro de los síntomas que más han analizado los estudios $3,7,10,12,19-21$ ha sido el insomnio y la calidad el sueño de los profesionales sanitarios, como también se tuvo presente en las cartas a los editores de Xu et al. ${ }^{16}$ y de Du et al. ${ }^{17}$.

Para realizar estos análisis, los autores de los artículos se han basado en los resultados ofrecidos por tres escalas validadas: el Índice de Gravedad del Insomnio (Insomnia Severity Index, ISI); el Índice de Calidad del Sueño de Pittsburg (Pittsburg Sleep Quality Index, PSQI); y la Escala de Atenas de Insomnio (Athens Insomnia Scale, AIS).

En la muestra de médicos de cabecera observada por Amerio et al. ${ }^{7}$, la gravedad del insomnio, según los resultados de la escala ISI, fue mayor en los profesionales que presentaban síntomas depresivos (de $11,60 \pm 5,53$ puntos o «insomnio leve») que en los profesionales sin sintomatología depresiva (de 6,39 \pm 5,11 puntos o «sin insomnio»). También Zhang et al. ${ }^{19}$, a través de la escala ISI, analizaron el insomnio en una muestra de 1563 profesionales sanitarios de hospitales de Wuhan, China, detectando una prevalencia de síntomas de insomnio del 36,1\%. Los profesionales de la salud de China, con una muestra representativa de 2186, entre los que se encontraban profesionales sanitarios (médicos y enfermeras) y 
profesionales sanitarios no médicos, a través de la misma escala de gravedad del insomnio (ISI), observaron una prevalencia del insomnio del $38,4 \%$ y del $30,5 \%$, respectivamente, siendo esta una diferencia estadísticamente significativa entre los dos grupos. Lai et al. ${ }^{3}$ también estudiaron el sueño de 1830 trabajadores sanitarios a través de la herramienta ISI, los cuales presentaron una prevalencia del $34 \%$, siendo las enfermeras las que mostraron peores valores en los resultados de la escala. En el estudio de Xiao et al..$^{20}$, aunque no presenta resultados de prevalencia del insomnio en el análisis de una muestra de 222 sanitarios que respondieron a la escala PSQI, sí afirma, a través de un análisis de correlación, que la combinación de estrés, ansiedad y autoeficacia del personal sanitario actúa sobre la calidad del sueño. Finalmente, Qi et al. ${ }^{21}$, en su estudio del sueño en una población china de 1306 profesionales de la salud, diferenciados entre profesionales de primera línea y profesionales que no estuvieron en primera línea, encuentran, a través de la encuesta con la escala PSQI, diferencias estadísticamente significativas entre los grupos (de 9,3 $\pm 3,8$ y 7,5 \pm 3 puntos, respectivamente); $y$, a través de la escala AIS, observan nuevamente diferencias estadísticamente significativas entre los profesionales sanitarios de primera línea (6,9 \pm 4 puntos) y los que no lo estuvieron (5,3 $\pm 3,8$ puntos).

\section{Factores de riesgo}

Los cuatro impactos psicológicos más importantes en los artículos analizados se asocian a diferentes factores de riesgo. Fundamentalmente, podemos destacar como factores de riesgo comunes: la edad, ser mujer, ser enfermera, el estado civil, la experiencia laboral,

\section{Soporte emocional en estas situaciones para aumentar la resiliencia de los profesionales sanitarios.}

tener hijos, la convivencia con personas mayores, las enfermedades crónicas concomitantes, el entorno en el que vive, los años de educación, la experiencia laboral, estar expuesto directamente a pacientes con COVID-19 o no, tener experiencia en el manejo de enfermedades infecciosas, la percepción de la utilización de equipos de protección personal (EPP) poco adecuados, la preocupación por poner en peligro la vida, la calidad de vida, el insomnio, la ansiedad y presentar síntomas depresivos $3,6,7,10-13,15,17,19-21,23$.

\section{LIMITACIONES}

A pesar de que la literatura médica incluida en este artículo ha contribuido al conocimiento del impacto psicológico en los profesionales sanitarios, se han identificado ciertas limitaciones y falta de datos. Primero, el uso de varios instrumentos estandarizados para analizar los mismos conceptos; por lo tanto, esto dificulta el poder unificar de forma universal los datos obtenidos. Segundo, los artículos obtenidos provienen en gran parte de China, por lo que se sugiere realizar más estudios en distintos países y continentes para poder comparar los resultados. Tercero, el tamaño de las muestras en los estudios analizados es muy heterogéneo, dificultando, así, la interpretación de los datos obtenidos. Cuarto, pese a usar un enfoque amplio en la búsqueda e identificación de estudios, nosotros solo hemos encontrado artículos en inglés. Finalmente, los estudios no tienen en cuenta el tiempo de exposición del personal sanitario a la situación de COVID-19, por lo que no sabemos si los síntomas detectados se mantienen, se agravan o disminuyen con el tiempo.

\section{CONCLUSIONES}

El impacto psicológico que ha producido la pandemia de la COVID-19 en los profesionales sanitarios es importante y abarca diferentes esferas, siendo necesario no solo su detección a través de instrumentos 
estandarizados, sino también su tratamiento para evitar posibles problemas a medio-largo plazo. Los profesionales sanitarios desempeñan un papel fundamental en la atención de los pacientes; por ello, se debería tener en cuenta a todos ellos, tanto a los que se encuentran en contacto directo con pacientes con COVID-19 en los medios hospitalarios o la denominada "primera línea», como a aquellos sanitarios que se encuentran en un medio comunitario. Asimismo, no podemos dejar de mencionar la importancia que tiene el poder ofrecer soporte emocional en estas situaciones para aumentar la resiliencia de los profesionales sanitarios.

Contribuciones de los autores: los autores han participado de igual manera en todos los procesos para la elaboración de este artículo de revisión. Todos los autores han leído y aceptado la publicación de este manuscrito.

Financiación: esta investigación no recibió fondos externos de ninguna agencia pública, comercial o sin fines de lucro.

Conflicto de intereses: los autores de este artículo manifiestan no tener ningún conflicto de intereses.

\section{BIBLIOGRAFÍA}

1. World Health Organization. Coronavirus disease (COVID-19) situation reports [Internet]. Disponible en: https://www.who.int/emergencies/diseases/novel-coronavirus-2019/situation-reports

2. Equipo COVID-19. Red Nacional de Vigilancia Epidemiológica (RNVE). Análisis de los casos de COVID-19 notificados a la RENAVE hasta el 10 de mayo en España [Internet]. Informe n. ${ }^{\circ} 33.29$ de mayo de 2020. Disponible en: https://www.isciii.es/QueHacemos/Servicios/VigilanciaSaludPublicaRENAVE/EnfermedadesTransmisibles/Documents/INFORMES/Informes $\% 20$ COVID-19/Informe\%20n\% C2\%BA \% 2033. \% 20 An\%C3\%A1lisis\%20de\%20los\%20casos\%20de\%20 COVID-19\%20hasta\%20el\%2010\%20de\%20mayo\%20 en \% 20Espa\%C3\%B 1a\%20a\%2029\%20de\%20 mayo\%20de\%202020.pdf

3. Lai J, Ma S, Wang Y, Cai Z, Hu J, Wei N, et al. Factors associated with mental health outcomes among health care workers exposed to coronavirus disease 2019. JAMA Netw Open. 2020;3(3):e203976.
4. Gold JA. Covid-19: adverse mental health outcomes for healthcare workers. BMJ. 2020;369:m1815.

5. Sun D, Yang D, Li Y, Zhou J, Wang W, Wang Q, et al. Psychological impact of 2019 novel coronavirus (2019$\mathrm{nCoV}$ ) outbreak on health workers in China. Epidemiol Infect. 2020;148:e96.

6. Zhu Z, Xu S, Wang H, Liu Z, Wu J, Li G, et al. COVID-19 in Wuhan: immediate psychological impact on 5062 health workers. medRxiv. 2020.02.20.20025338.

7. Amerio A, Bianchi D, Santi F, Costantini L, Odone A, Signorelli C, et al. Covid-19 pandemic impact on mental health: a web-based cross-sectional survey on a sample of Italian general practitioners. Acta Biomed. 2020;91(2): 83-8.

8. Ko NY, Lu WH, Chen YL, Li DJ, Wang PW, Hsu ST, et al. COVID-19-related information sources and psychological well-being: an online survey study in Taiwan. Brain Behav Immun. 2020;87:153-4.

9. Wu Y, Wang J, Luo C, Hu S, Lin X, Anderson AE, et al. A comparison of burnout frequency among oncology physicians and nurses working on the frontline and usual wards during the COVID-19 epidemic in Wuhan, China. J Pain Symptom Manage. 2020;60(1):e60-e65.

10. Zhang WR, Wang K, Yin L, Zhao WF, Xue Q, Peng M, et al. Mental health and psychosocial problems of medical health workers during the COVID-19 epidemic in China. Psychother Psychosom. 2020;89:242-50.

11. Liu Z, Han B, Jiang R, Huang Y, Ma C, Wen J, et al. Mental health status of doctors and nurses during $\mathrm{CO}$ VID-19 epidemic in China. SSRN Electron J. 2020. [Preprint]. Disponible en: https://ssrn.com/abstract=3551329

12. Pappa S, Ntella V, Giannakas T, Giannakoulis VG, Papoutsi E, Katsaounou P. Prevalence of depression, anxiety, and insomnia among healthcare workers during the COVID-19 pandemic: a systematic review and meta-analysis. Brain Behav Immun. 2020;S08891591(20)30845-X. [En prensa].

13. Guo J, Liao L, Wang B, Li X, Guo J, Tong Z, et al. Psychological effects of COVID-19 on hospital staff: a national cross-sectional survey of China Mainland. SSRN Electron J. 2020. [Preprint]. Disponible en: https://ssrn. com/abstract $=3550050$

14. Tan BYQ, Chew NWS, Lee GKH, Jing M, Goh Y, Yeo LLL, et al. Psychological impact of the COVID-19 pandemic on health care workers in Singapore. Ann Intern Med. 2020;M20-1083. [En prensa].

15. Lu W, Wang H, Lin Y, Li L. Psychological status of medical workforce during the COVID-19 pandemic: a crosssectional study. Psychiatry Res. 2020;288:112936.

16. Xu J, Xu QH, Wang CM, Wang J. Psychological status of surgical staff during the COVID-19 outbreak. Psychiatry Res. 2020;288:112955. 
17. Du J, Dong L, Wang T, Yuan C, Fu R, Zhang L, et al. Psychological symptoms among frontline healthcare workers during COVID-19 outbreak in Wuhan. Gen Hosp Psychiatry. 2020;S0163-8343(20)30045-1. [En prensa].

18. Wu D, Jiang C, He C, Li C, Yang L, Yue Y. Stressors of nurses in psychiatric hospitals during the COVID-19 outbreak. Psychiatry Res. 2020;288:112956.

19. Zhang C, Yang L, Liu S, Ma S, Wang Y, Cai Z, et al. Survey of insomnia and related social psychological factors among medical staff involved in the 2019 novel coronavirus disease outbreak. Front Psychiatry. 2020;11:306.

20. Xiao H, Zhang Y, Kong D, Li S, Yang N. The effects of social support on sleep quality of medical staff treating patients with coronavirus disease 2019 (COVID-19) in January and February 2020 in China. Med Sci Monit. 2020;26:e923549.

21. Qi J, Xu J, Li BZ, Huang JS, Yang Y, Zhang ZT, et al. The evaluation of sleep disturbances for Chinese frontline medical workers under the outbreak of COVID-19. Sleep Med [Internet]. 2020;72:1-4. Disponible en: https:// linkinghub.elsevier.com/retrieve/pii/S1389945720302264

22. Liu CY, Yang YZ, Zhang XM, Xu X, Dou QL, Zhang WW, et al. The prevalence and influencing factors in anxiety in medical workers fighting COVID-19 in China: a crosssectional survey. Epidemiol Infect. 2020;148:e98.

23. Temsah MH, Al-Sohime F, Alamro N, Al-Eyadhy A, AlHasan K, Jamal A, et al. The psychological impact of COVID-19 pandemic on health care workers in a MERSCoV endemic country. J Infect Public Health [Internet]. 2020;13(6):877-82.

24. Rolim Neto ML, Gomes Almeida H, Esmeraldo JD, Bezerra Nobre C, Rodrigues Pinheiro W, Tavares de Oliveira $\mathrm{CR}$, et al. When health professionals look death in the eye: the mental health of professionals who deal daily with the 2019 coronavirus outbreak. Psychiatry Res. 2020;288:112972.

25. Mo Y, Deng L, Zhang L, Lang Q, Liao C, Wang N, et al. Work stress among Chinese nurses to support Wuhan in fighting against COVID-19 epidemic. J Nurs Manag. 2020;10.1111/jonm.13014. [En prensa].

26. Frías CE, Cuzco C, Frías Martín C, Pérez-Ortega S, Triviño López JA, Lombraña M. Resilience and emotional support in health care professionals during the COVID-19 pandemic. J Psychosoc Nurs Ment Health Serv. 2020; 58(6):5-6. 